\title{
Web 6.0: Journey From Web 1.0 To Web 6.0
}

\author{
Özgür Önday
}

Department of Business Administration, Yeditepe University, Istanbul, Turkey

Abstract
The fast track toward the improvement of Web is instituted to be as an out and out wonder in the present society with
fused utilization of current creative innovation and rethinking the method for arranging, imparting and working togeth-
er with person which in wording lead us to blend of terrific victories and disappointments. The motivation behind this
paper is to comprehend and conceptualize the development of Web from the scratch to the up and coming patterns in
the field of Web Technology.
The World Wide Web as the biggest data build has had much progress since its approach. This paper gives a foundation
of the development of the web from web 1.0 to web 4.0 . Web 1.0 as a snare of data associations, Web 2.0 as a trap of
individuals associations, Web 3.0 as a trap of learning associations and web 4.0 as a trap of knowledge associations are
portrayed as four ages of the web in the paper. Additionally, in this work, we inquire about the advancement of things to
come of the (Web 5.0 and Web 6.0). Likewise, we present the present status and worries about the Web as a data source
and correspondence channel.

Corresponding author: Özgür Önday, Department of Business Administration, Yeditepe University, Istanbul, Turkey. Tel: +0216 57800 00; E-mail: ozgur.onday@std.yeditepe.edu.tr

Keywords: Web Generations; Web 1.0; Web 2.0; Web 3.0; Web 4.0; Web 5.0; Web 6.0; World Wide Web; WWW; Semantic Web; WebOS; Intelligent Agent.

Received: November: 21, 2019, Accepted: December: 02, 2019, Published: December: 06, 2019

\section{Introduction}

In the present period Web Technology can be effectively characterized by the client in various graphic manners. Yet, matter in certainty numerous client are very obscure to the data that from where the WWW was instituted first. As this paper express the development of Web so it is essential to start the story from the earliest starting point where it was expressed first.

Web was presented by Tim Burners-Lee in late 1989. His perspective on the abilities of the World Wide Web was communicated by three advancements, ordinarily connected with three stages: specifically, the Web of reports (Web 1.0), the Web of individuals (Web 2.0) and the Web of information (the still-to-be-acknowledged Web 3.0). Through its life cycle, the World Wide Web has experienced different periods of improvement. Passing by the pattern of steady advancement, the Web is currently gradually traveling to more information driven stage with regards to Web variant 3.0. 
Citation: Özgür Önday (2019) Web 6.0: Journey From Web 1.0 To Web 6.0. Journal of Media \& Management.

SRC/JMM-102. DOI: doi.org/10.47363/JMM/2019(1)102

- Web 1.0 is the original of the web which as per Berners-Lee, could be viewed as the read-just web and furthermore as an arrangement of comprehension. Web 1.0 started as a data place for organizations to communicate their data to individuals. The early web gave constrained client cooperation or substance commitments and just permitted to look through the data and read it.

Web 2.0 was characterized by Dale Dougherty in 2004 as a read-compose web. The advances of web 2.0 permit gathering and overseeing huge worldwide groups with regular interests in social connections. The contrasts between web 1.0 and web 2.0 are various that are depicted in the token of the paper.

Web 3.0 or semantic web wants to diminish human's assignments and choices and leave them to machines by giving machine-comprehensible substance on the web. In General, web 3.0 is incorporated two principle stages, semantic advances and social registering condition. The semantic advancements speak to open measures that can be applied on the highest point of the web. The social registering condition permits human-machine co-activities and sorting out an enormous number of the social web networks.

Web 4.0 will be as a perused compose executionsimultaneousness web with wise connections, however there is still no careful meaning of it. Web 4.0 and Web 5.0 is otherwise called advantageous web in which human personality and machines can communicate in beneficial interaction.

This paper is organized so that, arranging acquiring nature of Web 1.0 and anticipating imminent qualities of Web 2.0 with included various components of the Web 3.0 semantic structures, while its degree is coordinated to investigate a more grounded thankfulness into building establishments of the up and coming age of Web 4.0 of Web applications. Likewise, in this work, we inquire about the advancement of things to come of the (Web 5.0 and Web 6.0). Likewise, we present the present status and worries about the Web as a data source and correspondence channel. This paper would attempt to build a user centric view of the composition of features that would be expected to be incorporated in future generations of Web technology. In sum, the paper presents a holistic view of the World Wide Web.

\section{Web 1.0}

In 1989, Tim Burners-Lee proposed making a worldwide hypertext space in which any system open data would be alluded to by a solitary Universal Document Identifier (UDI). The fantasy behind of the web was to make a typical data space in which individuals impart by sharing data.

Web 1.0 was for the most part a read-just web. Web 1.0 was static and to some degree mono-directional. Organizations could give inventories or pamphlets to show their preparations utilizing the web and individuals could peruse them and reached with the organizations. In reality, the inventories and the leaflets were correspondingly ads in papers and magazines and most proprietors of web based business sites utilized shopping basket applications in various shapes and structures. The sites included static HTML pages that refreshed inconsistently. The primary objective of the sites was to distribute the data for anybody whenever and build up an online nearness. The sites were not intelligent and undoubtedly were as pamphlet product. Clients and guests of the sites could just visit the locales with no effects or commitments and connecting structure was excessively feeble. Center conventions of web 1.0 were HTTP, HTML and URI.

\section{WEB 2.0}

The idea of Web 2.0 was begat by Dale Dougherty, web pioneer and O'Reilly VP in 2004 with a gathering meeting to generate new ideas among O'Reilly and MediaLive International. Web 2.0 is additionally called the intelligence Web, individuals driven Web, participative Web, and read/compose Web.

With Web 2.0 we moved away from a conventional model of distributers making content accessible to customers, to a significantly more unique participatory model where most of website page engineers had the 
Citation: Özgür Önday (2019) Web 6.0: Journey From Web 1.0 To Web 6.0. Journal of Media \& Management.

SRC/JMM-102. DOI: doi.org/10.47363/JMM/2019(1)102

chance to refresh their own media-rich sites as regularly as they preferred. Data started to stream in the two bearings between substance suppliers and watchers. For instance, hit counters generally show Web sites relative fame, while the volume of client remarks gives a proportion of client investment.

This was a time of client produced content and enormous internet based life communication., blogging, video sharing, visiting, facilitated administrations, web applications, voice over IP, messages, texts, social bookmarking, podcasting, picture sharing, weblogs, mashups and folksonomies, and so on and a wide range of online intelligence ended up conceivable and has demonstrated to be an extraordinary achievement. The beneath figure delineates different applications and advances dependent on Web 2.0 age.

The greatest endowment of Web 2.0 to the humankind is web based life locales for example Facebook, Twitter, Linked-In, Zynga, Google+, Flickr, Foursquare, and You-Tube, and so forth. The web based life has associated individuals universally in manners unfathomable only a couple of decades back.

One of the key drivers of the improvement of Web 2.0 is the rise of another age of Web-related innovations and measures. Ajax, JavaScript, Cascading Style Sheets (CSS), Document Object Model (DOM), Extensible HTML (XHTML), XSL Transformations (XSLT)/XML, and Adobe Flash furnished clients with a rich and fun intelligent experience. These advances show and convey Web benefits simply like work area programming, making disseminated preparing troubles undetectable.

The primary advances and administrations of web 2.0 are incorporated web journals, extremely basic syndication (RSS), wikis, mashups, labels, folksonomy, and label mists that some of them portrayed as follows in quickly:

- Blogs-The term weblog (or blog) was proposed by Jorn Barger in 1997. The blog is incorporated the website pages called posts which distributed sequentially with the latest first, in diary style. Guests of the web journals can include a remark beneath a blog passage. Most sites are printed and yet there are different sorts, for example, photoblogs or photologs, videoblogs or video blogs and digital recordings.
- Posts of online journals can be labeled with watchwords so as to arrange the subjects of the posts. For example when the post ends up old, it tends to be documented into a standard, subject based menu framework. Connecting is another significant part of blogging. Connecting extends on the conversational idea of the blogosphere and its feeling of instantaneousness and encourages recovery and to reference data on various sites.

- Really Simple Syndication - RSS is a group of web channel arrangements utilized for syndicating content from websites or site pages. RSS is a XML document that abridges data things and connections to the data sources. Utilizing RSS, clients are educated regarding updates of the websites or sites which they're keen on. Particle is another syndication particular planned for settling issues of various incongruent RSS variants.

- Wikis-A wiki is a site page (or set of site pages) that can be effectively altered by any individual who is permitted get to. In contrast to online journals, past forms of wikis can be analyzed by a history work and can be reestablished by a rollback work. Wiki highlights are incorporated: wiki markup language, straightforward site structure and route, basic layout, supporting of various clients, worked in hunt highlight and basic work process.

- Mashups-Web mashup is a site page (or site) that joins data and administrations from different sources on the web. Mashups can be assembled into seven classifications: mapping, search, versatile, informing, sports, shopping, and motion pictures. In excess of 40 percent of mashups are mapping mashups. It is simpler and snappier to make mashups than to code applications without any preparation in customary manners; this ability is one of most significant highlights of web 2.0. Mashups are by and large made utilizing application programming interfaces. Several development tools are available to create blogs, wikis, mashups, and social networks. These tools, such as mashup tools, wiki engines, blog software, make adoption of web 2.0 easier, quicker, and cheaper. Developers use three basic development approaches to create applications of web 2.0: Asyn- 
Citation: Özgür Önday (2019) Web 6.0: Journey From Web 1.0 To Web 6.0. Journal of Media \& Management. SRC/JMM-102. DOI: doi.org/10.47363/JMM/2019(1)102

chronous JavaScript and XML (AJAX), Flex, and the Google Web Toolkit.

- Asynchronous JavaScript and XML-AJAX is a web improvement approach that utilized for advancement of most intuitive sites by recovering limited quantity of information from web server and show it on the web application without reloading the entire page [13]. AJAX is incorporated a few advancements: XHTML or HTML, falling templates (CSS), JavaScript and XML.

- Flex-Adobe Flex is a product advancement pack (SDK) to make and convey cross stage rich web applications (RIAs) on the web. Flex depends on Flash and supports normal structure designs by giving a programming language.

- Google Web Toolkit-GWT is an open source Java advancement system that makes making AJAX applications simple. It permits to web designers investigate AJAX applications in the Java language utilizing their preferred Java advancement apparatuses. GMT gives a complier and an uncommon internet browser that help engineers to investigate the GWT applications.

\section{Web 3.0}

John Markoff of the New York Times proposed web 3.0 as third era of the web in 2006. The fundamental thought of web 3.0 is to characterize structure information and connection them so as to increasingly compelling revelation, robotization, incorporation, and reuse crosswise over different applications. Web 3.0 attempts to connect, coordinate, and investigate information from different informational collections to acquire new data stream; It can improve information the executives, bolster availability of portable web, mimic innovativeness and development, empower factor of globalization marvels, upgrade clients' fulfillment and help to sort out joint effort in social web.

Web 3.0 is otherwise called semantic web. Semantic web was concocted by Tim Berners-Lee, creator of the World Wide Web. There is a committed group at the World Wide Web consortium (W3C) attempting to improve, broaden and institutionalize the framework, dialects, distributions and apparatuses have just been created. Semantic web is a web that can exhibit things in the methodology which PC can get it. The fundamental significant motivation behind semantic web is to make the web meaningful by machines and not just by people.

The present web is a snare of reports, somehow or another like a worldwide record framework that the most significant issues about it is incorporated: The trap of archives was intended for human utilization in which essential articles are reports and connections are between reports (or parts of them). Semantics of substance and connections are verifiable and the level of structure between items is genuinely low.

Semantic web is being to be created to beat the issues of current web. Semantic Web can be characterized a trap of information, here and there like a worldwide database that most its highlights are incorporated: The point of configuration web of information is machines first, people later. Essential articles are things so connections are between things. Semantics of substance and connections are express and the level of structure between items is high founded on RDF model.

The primary distinction between web 2.0 and web 3.0 is that web 2.0 focuses on substance inventiveness of clients and makers while web 3.0 focuses on connected informational indexes.

The layers of the semantic web architecture are briefly described as follows:

- Unicode and URI: Unicode is utilized to speak to of any character extraordinarily whatever this character was composed by any language and Uniform Resource Identifier (URI) are novel identifiers for assets of various types. The usefulness of Unicode and URI could be portrayed as the arrangement of a novel distinguishing proof instrument inside the language stack for the semantic web.

- Extensible Markup Language: XML and its related models, for example, namespaces (NS), and patterns are utilized to shape a typical way to structure information on the web with no correspondence between the implications of the information. XML is utilized as a base linguistic structure for different innovations created for the upper layers of the semantic web. NS is utilized to recognize and recognize distinctive XML components of various vocabularies. It supports blending of various components from different vocabularies to do a particular capacity. XML blueprint guarantees that the got data is as 
Citation: Özgür Önday (2019) Web 6.0: Journey From Web 1.0 To Web 6.0. Journal of Media \& Management. SRC/JMM-102. DOI: doi.org/10.47363/JMM/2019(1)102

per the sent data when two applications at this level trade data with together.

- Resource Description Framework: RDF is a straightforward information model that utilizations URIs to recognize online assets and depicts connections between the assets regarding named properties and qualities. By and large, the RDF family bolsters interoperability at the semantic level. RDF improvements comprise of the base web language, with the goal that specialists can make consistent derivations to perform capacities dependent on metadata.

- RDF Schema: gives a predefined, essential sort framework for RDF models. It portrays classes and properties of the assets in the fundamental RDF model. RDF Schema gives a straightforward thinking system to induce kinds of assets.

- - Ontology: The metaphysics layer depicted properties and the connection among properties and unique. Metaphysics can be characterized as a gathering of terms used to portray a particular area with the capacity of surmising.

- Logic and Proof: This layer is over the cosmology structure to make new inductions by a programmed thinking framework. The specialists can make conclusions about whether specific assets fulfill their prerequisites by utilizing such the thinking frameworks.

- - Trust: The last layer of the stack addresses trust in order to provide an assurance of quality of the information on the web and a degree of confidence in the resource providing this information.

\section{Web 4.0 and Future Web}

Web 4.0 can be considered as a Ultra-Intelligent Electronic Agent, cooperative web and Ubiquitous web. Connection among people and machines in advantageous interaction was intention behind of the harmonious web. Ground-breaking as human cerebrum, progress in the improvement of broadcast communications, headway on nanotechnology on the planet and controlled interfaces utilizing web 4.0. In basic words, machines would be sharp on perusing the substance of the web, and respond through executing and choosing what to execute first to stack the sites quick with predominant quality and execution and fabricate all the more ordering interfaces.
Web 4.0 will be perused compose simultaneousness web. It guarantees worldwide straightforwardness, administration, dissemination, interest, cooperation into key networks, for example, industry, political, social and different networks. WebOS will be, for example, a middleware in which will begin working like a working framework. WebOS will be parallel to the human cerebrum and suggests a monstrous snare of profoundly insightful communications.

\section{Web 5.0}

Web 5.0 is as yet an underground thought in advancement and there is no precise meaning of how it would be. Web 5.0 can be considered as Symbiotic web, decentralized for example it is unimaginable to expect to have a Personal Server (PS) for any close to home information or data put away on the net, and individuals attempts to get interconnected by means of Smart Communicator (SC), like Smart telephones, Tablets or Personal Robots for example is spoken to as its very own symbol inside the SC, that will have the option to surf alone in the 3D Virtual universe of the Symbiotic. The Symbiotic servers will have the option to utilize a piece of "memory and estimation control" of each interconnected SC, so as to figure the billions and billions required information to fabricate the $3 \mathrm{D}$ world, and to encourage its Artificial Intelligence surf alone. At present the Web is "inwardly" unbiased: don't feel the client sees. The organization Emotive Systems has made neurotechnology through earphones that enable clients to associate with substance that meets their feelings or change progressively outward appearance a "symbol".

Paper thinks about the fifth era (Web 5.0) as a "Tactile Emotive Web." Web 5.0 considers the sentiments of the client. It is guided by advancements that as of now exist to quantify sentiments and their belongings. For instance, an organization called Emotiv Systems 2 works in the field of neurotechnology. With earphones, the human cerebrum can speak with a machine. The machine can peruse cognizant musings, feelings, outward appearances and head revolutions. Research in depicts Web 5.0 as a "Symbionet Web." They notice it is "inwardly" unbiased and takes note of that it doesn't depend on a client's sentiments. In this respects, creator of referenced that individuals "feel" and "think" and they don't really act sensibly. We figure it would consider 
Citation: Özgür Önday (2019) Web 6.0: Journey From Web 1.0 To Web 6.0. Journal of Media \& Management.

SRC/JMM-102. DOI: doi.org/10.47363/JMM/2019(1)102

organized information alongside organized archives. Likewise, Web 5.0 may exploit information combination calculations and applications to converge with pervious Web pages.

The information combination field has just demonstrated effective in various areas, including revelation science and business insight, and it might have the option to work with multiagent frameworks. We imagine that Web 5.0 is about "Combination Web" where machines and individuals will process information in structures that they can manage, connect with, and settle on choices with. The Web 5.0 is normal not just about helping people to be better at the things we would already be able to do; the Web 5.0 is conceivably about helping people to do things which we can't as of now do. A relationship is 200 years back, individuals could move really quick, for instance, in a train, yet we couldn't fly. As of now, individuals can think well (as we as of now get suspecting), however in the event that the Web 5.0 moves toward becoming what it appears it could turn out to be, at that point our reasoning may take a bounce equivalent to going from hurrying to flying. Almost certainly the Web 5.0 won't become all that we may dream that it could turn out to be, similarly as AI has not turned into every one of that individuals figured it may turn into. In any case, if the Web 5.0 turns out to be simply part of what we figure it may progress toward becoming, and after that it may prompt things which we right now experience serious difficulties envisioning. We may see Web 5.0 or "Combination Web" join Web for all to help individuals with uncommon needs. Web 5.0 could be a perused composes execute-simultaneous combination Web.

Web 5.0 is relied upon to be based on the intensity of Semantic "Web 3.0" and Symbolic "Web 4.0.".

\section{Web 6.0}

Another hub named web Service Extensions has been added to the Internet Information Services Manager (ISM) in Internet Information Services (IIS) 6.0. Web administration augmentations are programs that expand the essential IIS usefulness of serving static substance. Instances of Web administration expansions are, Active Server Pages (ASP), ASP.NET, FrontPage Server Extensions, Server-side incorporates (SSI), Internet Database Connector, Web Distributed Authoring and Versioning (Web DAV), Common
Gateway Interface (CGI), Internet Server API (ISAPI), Active Server Pages (ASP), ASP.NET, FrontPage Server Extensions, Server-side incorporates (SSI), Database Connector, Web Distributed Authoring and Versioning (Web DAV), Common Gateway Interface (CGI), and Internet Server API (ISAPI).

Web Information Services (IIS) 6.0 conveys Web facilitating administrations through a flexible design that you can use to oversee server assets with improved solidness, productivity, and execution. IIS isolates applications into confined pools and consequently distinguishes memory releases, imperfect procedures, and over-used assets. At the point when issues happen, IIS oversees them by closing down and redeploying flawed assets and interfacing defective procedures to systematic apparatuses.

\section{Conclusion}

Taking everything into account, change from Web 1.0 to Web 2.0 is discretionary, as it relies upon the need to have a powerful site. Or maybe, we think transformation from Web 1.0 or Web 2.0 to Web 3.0 is important to utilize for future improvement and progression of the Web. In this respects, a few arrangements have been proposed to help in arriving at Webs (e.g., RD$\mathrm{FaCE}$ ). In the following (Web 4.0) or harmonious Web, information is relied upon to be organized well.

Therefore, ontologies will be the new gold; namely, ending up with an enriched and efficient conceptualization analysis of a specific area of interest. This will lead to high knowledge sharing over databases and documents on the Web. Therefore, agent-based applications will be able to intelligently reason and perform tasks based on metadata. Moreover, experts in the semantic Web foresee that intelligent software agents will be interacting with humans in symbiosis. Thus, the interplay between Web 5.0 and Web 6.0 will be a key technology for the growth of knowledge and for making the Web more useful for humanity. 